\title{
Optimising Micro-Hydroxyapatite Reinforced Poly(lactide acid) Electrospun Scaffolds for Bone Tissue Engineering
}

\author{
Muna M. Kareem and K. Elizabeth Tanner", \\ Biomedical Engineering Division, James Watt School of Engineering, University \\ of Glasgow, Glasgow, G12 8QQ, UK \\ \# Now at School of Engineering and Materials Science and Institute of \\ Bioengineering, Queen Mary University of London, Mile End Road, London, E1 \\ 4NS, UK
}

ORCiD IDs

Muna M Kareem https://orcid.org/0000-0002-3889-8309

K Elizabeth Tanner https://orcid.org/0000-0003-2257-0218

\section{Graphic Abstract}
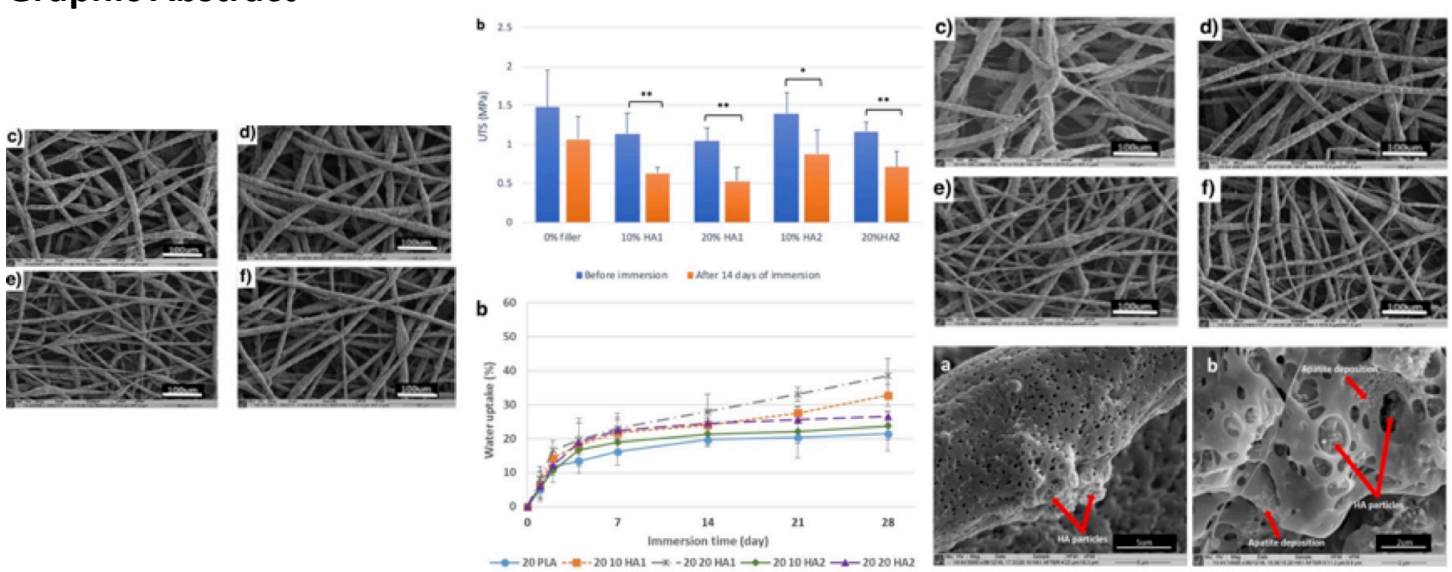

\section{Corresponding Author:}

\section{K.E. Tanner}

School of Engineering and Materials Science,

Queen Mary University of London,

Mile End Road

London,

E1 4NS,

UK

Phone: +44-20-78826785

e-mail: $\quad$ k.e.tanner@qmul.ac.uk 


\begin{abstract}
HA-mineralised composite electrospun scaffolds have been introduced for bone regeneration due to their ability to mimic both morphological features and chemical composition of natural bone ECM. Micro-sized HA is generally avoided in electrospinning due to its reduced bioactivity compared to nano-sized HA due to the lower surface area. However, the high surface area of nanoparticles provides a very high surface energy, leading to agglomeration. Thus, the probability of nanoparticles clumping leading to premature mechanical failure is higher than for microparticles at higher filler content. In this study, two micron-sized hydroxyapatites were investigated for electrospinning with PLA at various contents, namely spray dried HA (HA1) and sintered HA (HA2) particles to examine the effect of polymer concentration, filler type and filler concentration on the morphology of the scaffolds, in addition to the mechanical properties and bioactivity. SEM results showed that fibre diameter and surface roughness of 15 and $20 \mathrm{wt} \%$ PLA fibres were significantly affected by incorporation of either HA. The apatite precipitation rates for HA1 and HA2-filled scaffolds immersed in simulated body fluid (SBF) were similar, however, it was affected by the fibre diameter and the presence of HA particles on the fibre surface.

Degradation rates of HA2-filled scaffolds in vitro over 14 days was lower than for HA1filled scaffolds due to enhanced dispersion of HA2 within PLA matrix and reduced cavities in PLA/HA2 interface. Finally, increasing filler surface area led to enhanced thermal stability as it reduced thermal degradation of the polymer.
\end{abstract}

Keywords:

Electrospinning, hydroxyapatite, polylactic acid, composite, micro-filler, bioactivity

\title{
1 INTRODUCTION
}

Electrospinning is a simple, versatile and cost effective technique to produce micro to nanoscale diameter fibres through the application of a high static voltage to a viscous polymer solution in a volatile solvent [1]. For bone tissue engineering, polymeric scaffolds are usually reinforced with bioceramics, including hydroxyapatite, to improve the bioactivity as well as enhance cell growth and response [2-5]. Most studies prefer to use nano-sized HA needle shaped fillers due to their high aspect ratio and uniform suspension as well as mimicking the natural bone structure better. However, Peng et al. [6] reported that needle-like micro-HA electrospun scaffolds lead to higher cell proliferation and differentiation in aligned scaffolds at the early culture stages than nano-HA in PLLA-HA. In another study, Tetteh et al. [7] electrospun nano and micro-size HA particles with polyether-urethane (PU) polymers (Z3A1 and Z9A1) and investigated the effect of particle size on fibre morphology, mechanical properties, biocompatibility, extracellular and calcified matrix production over a 28 day period. Their results revealed that inclusion of both sizes of HA particles in PU-HA solutions reinforced the scaffolds and increased the mechanical properties, although for Z9A1, both yield strength and Young's modulus of micro-HA containing scaffolds were significantly higher than those of nano-HA containing scaffolds. Hence, further study on the effects of using micro-sized HA on scaffold properties is important.

This paper examines the effect of increasing HA specific surface area and using microsized HA at higher filler levels on the porosity, bioactivity, thermal stability and mechanical properties of the electrospun PLA composites. 


\section{MATERIALS AND METHODS}

\subsection{Materials}

Polylactic acid (Ingeo Biopolymer 3001D PLA) with an average molecular weight (Mw) of $136000 \mathrm{~g} \mathrm{~mol}^{-1}$ [8], $1.5 \%$ D-lactide content, and density of $1.24 \mathrm{~g} \mathrm{~cm}^{-3}$ was supplied by NatureWorks LLC (Minnetonka, USA). Two types of micron sized-hydroxyapatite fillers were used: spray dried hydroxyapatite (HA1) in the form of agglomerates of nanoscale needles (P218R, $d_{50}=4.02 \mu \mathrm{m}$, specific surface area $=13.536 \mathrm{~m}^{2} \mathrm{~g}^{-1}$ ) and a solid, previously sintered hydroxyapatite (HA2) with smooth surfaces (P220S, $d_{50}=3.59$ $\mu \mathrm{m}$, specific surface area $\left.=0.965 \mathrm{~m}^{2} \mathrm{~g}^{-1}\right)$. Both types of hydroxyapatite have a theoretical density of $3.162 \mathrm{~g} \mathrm{~cm}^{-3}$ and were purchased from Plasma Biotal Ltd, UK. Chloroform (99.8+\%, certified AR for analysis) was supplied by Fisher Scientific, UK.

\subsection{Electrospinning solutions preparation}

15 and $20 \%$ (wt/v) PLA solutions were prepared by dissolving PLA in chloroform. The container was sealed to prevent solvent evaporation and left to stir overnight at room temperature to obtain homogenous mixtures. Once the PLA appeared to have dissolved completely, HA1 or HA2 was added to the solution to give final compositions of 10 or 20 vol\% (22.1 or 38.9 wt\% respectively) in PLA. The PLA/filler solutions were stirred for 30 minutes, followed by ultrasonic agitation for at least 15 minutes to ensure that the filler was well distributed.

\subsection{Fabrication of scaffolds}

Electrospinning experiments were performed using a horizontally placed electrospinning setup. PLA/filler solutions were loaded into a $10 \mathrm{~mL}$ luer-lock glass syringe with a straight blunt end metal needle (16 gauge, $30 \mathrm{~mm}$ long). The samples were electrospun onto $75 \times 25 \mathrm{~mm}$ microscope slides at a flow rate of $1 \mathrm{~mL} / \mathrm{h}$ and voltage of $13.7 \mathrm{kV}$ with the needle tip to collector distance set at $20 \mathrm{~cm}$. After electrospinning, scaffolds were left at least 3 hours to ensure complete solvent evaporation. Non-filled PLA scaffolds were used as controls.

\subsection{Characterisation of electrospun scaffolds}

\subsubsection{Scanning Electron Microscopy (SEM)}

The morphology of the electrospun scaffolds was observed using scanning electron microscope (SEM, JOEL JSM-6400, Tokyo, Japan) at an accelerated voltage of $10 \mathrm{kV}$. Specimens were cut from each PLA and PLA/HA combination and mounted on aluminium stubs using double sided carbon conductive adhesive tape. The specimens were sputter coated with a thin layer of gold-palladium using a Quorum Q150T ES sputter coater. Coating was carried out at a current of $25 \mathrm{~mA}$ for 4 minutes maximum (stopping at $20 \mathrm{~nm}$ thickness). The average fibre diameter of each scaffold type was calculated by analysing at least 50 fibres from three different SEM images using Image J software (NIH, USA). The total porosity of the scaffolds was calculated using the gravimetric method reported previously [9-11]. The thickness of the scaffolds were measured and the total porosity calculated using equation 1 :

Scaffold porosity $(\%)=\left(1-\frac{\text { Scaffoldapparent density }\left(\mathrm{g} \mathrm{cm}^{-\mathbb{I}}\right)}{\text { Scaffold bulkdensity }\left(\mathrm{g} \mathrm{cm}^{-5}\right)}\right) \times 100 \%$

The apparent density of the scaffolds were calculated using equation 2 : 
Apparent density $\left(\mathrm{g} \mathrm{cm}^{-3}\right)=\frac{\text { scaffold mass }(\mathrm{g})}{\text { scaffold thickness }(\mathrm{cm}) \times \operatorname{scaff} \text { fold area }\left(\mathrm{cm}^{2}\right)} \quad$ Eqn 2

The bulk density for plain PLA scaffolds were taken as $1.24 \mathrm{~g} \mathrm{~cm}^{-3}$, while the bulk density of PLA/HA scaffolds were calculated using equation 3 [12]:

$\rho_{\text {scaffold }}=\left(m_{P L A}+m_{H A}\right) /\left(m_{P L A} / \rho_{P L A}+m_{H A} / \rho_{H A}\right)$

Eqn 3

Where $\rho_{\text {scaffold }}=$ bulk density of electrospun PLA/HA scaffold, $m_{H A}=$ mass of $\mathrm{HA}$ added to scaffold, $m_{P L A}=$ mass of PLA in the scaffold, $\rho_{P L A}=$ bulk density of PLA, and $\rho_{H A}=$ bulk density of HA $\left(3.162 \mathrm{~g} \mathrm{~cm}^{-3}\right)$.

\subsubsection{Biodegradation and bioactivity measurements}

In order to evaluate and compare the dissolution rate and bioactivity among the nonfilled and HA-containing PLA scaffolds, samples were immersed in corrected Simulated Body Fluid (c-SBF) for up to 28 days, with the solutions changed every 7 days [13]. cSBF was prepared following the procedure reported by Oyane et al. [14]. Five samples of each scaffold type were extracted at 7, 14, 21 and 28 days. After each degradation period, samples were taken out of the solution, washed thoroughly with distilled water, and then blotted gently with a paper towel to remove excess water after which they were immediately weighed with analytical balance. Water uptake percentage was calculated using equation 4 [15-17]:

Percentage of water uptake $=\frac{\mathrm{w}_{\text {wret }}-\mathrm{w}_{\text {dry }}}{\mathrm{w}_{\text {dry }}} \times 100$

Eqn 4

Where $W_{\text {wet }}$ is the wet weight of the sample, and $W_{\text {dry }}$ is the dry weight of the sample. After measuring the wet weights, samples were dried for $48 \mathrm{~h}$ in a desiccator and weighed again. Weight loss percentage was then calculated using equation $5[16,17]$ : Weight loss percentage $=\frac{\mathrm{W}_{\mathrm{s}}-\mathrm{W}_{T}}{\mathrm{~W}_{\mathrm{s}}} \times 100$

Eqn 5

Where $W_{0}$ is the initial weight of the sample and $W_{r}$ is the residual weight of the sample after degradation.

\subsubsection{Fourier Transform Infrared (FTIR) spectroscopy}

Fourier transform infrared (FTIR) spectra for non-filled and HA-filled PLA scaffolds before and after 14 and 28 days of immersion in C-SBF were recorded using a Spectrum One FTIR Spectrometer (PerkinElmer Inc., Waltham, MA, USA). Samples were tested directly using the Attenuated Total Reflectance (ATR) accessory. Scans were obtained in absorbance mode across the $4000-400 \mathrm{~cm}^{-1}$ wavenumber range at a resolution of $8 \mathrm{~cm}^{-1}$ with 16 scans performed per spectrum and analysed using Spectrum ${ }^{\circledR}$ software (PerkinElmer Inc., Waltham, MA, USA). Five samples were tested for each type of scaffold and the spectra averaged using KnowltAll ${ }^{\circledR}$ Academic Edition Spectroscopy Software (Bio-Rad Laboratories, Inc., Hercules, CA, USA).

\subsubsection{Mechanical testing}

The tensile properties of the electrospun scaffolds were measured before and after immersion using uniaxial tensile testing. Tensile test samples were cut to $10 \mathrm{~mm}$ by $60 \mathrm{~mm}$ to provide a $40 \mathrm{~mm}$ test gauge length (reduced size ISO 13934) and mounted 
on rectangular cardboard frames with outer dimensions of $60 \times 40 \mathrm{~mm}$ and an inner rectangular cut out at $40 \times 20 \mathrm{~mm}$ and fixed by adhesive tape to the frame. Prior to testing, the thickness of the samples were measured, the frames were then placed into the test machine and the sidewalls of the frame cut. A Zwick/Roell Z2.0 (Zwick Roell, Kennesaw, GA, USA) tensile test machine was used with a $5 \mathrm{~N}$ load cell operating

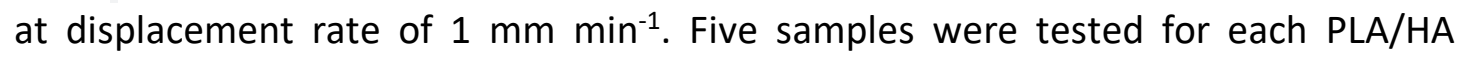
combination and the force-displacement data was collected for all the samples using Zwick/Roell TestXpert ${ }^{\circledR}$ software. The average values of Young's modulus (E) and ultimate tensile strength (UTS) were calculated.

\subsubsection{Thermogravimetric analysis (TGA)}

HA content in the PLA scaffolds and apatite deposition on the scaffold surfaces were measured by thermogravimetric analysis (TGA) using a TGA Q500 (TA Instruments, New Castle, DE, USA) under nitrogen atmosphere heating from 10 to $500^{\circ} \mathrm{C}$ at a rate of $20^{\circ} \mathrm{C} \mathrm{min}-1$. Two samples for each combination were tested. All the results were plotted as temperature versus weight loss and curves were analysed using Universal Analysis 2000 software (TA Instruments, New Castle, DE, USA).

\subsection{Statistical analysis}

Results are expressed as mean \pm standard deviation (SD) in all experiments. Statistical analysis was performed using a one- and two way analysis of variance (ANOVA) with p-values less than 0.05 considered statistically significant, and Student's t-test was used for pairwise comparisons.

\section{RESULTS}

\subsection{SEM and Porosity Measurements}

Figure 1 shows the morphologies of electrospun 15 and 20\% PLA fibres containing 0 and $20 \%$ of HA1 or HA2 before immersion in SBF. Non-filled 15 and 20\% PLA scaffolds exhibited uniform fibre diameter and relatively smooth surfaces with an average fibre diameters of $9.28 \pm 1.64 \mu \mathrm{m}$ for $15 \%$ PLA scaffolds and $11.16 \pm 1.66 \mu \mathrm{m}$ for scaffolds with $20 \%$ PLA concentration. However, introducing 10 or $20 \%$ of HA1 or HA2 into PLA resulted in non-uniform fibres with rough surfaces and enlarged diameters. The average fibre diameter, total porosity and apparent density values of the scaffolds are listed in Table 1. 

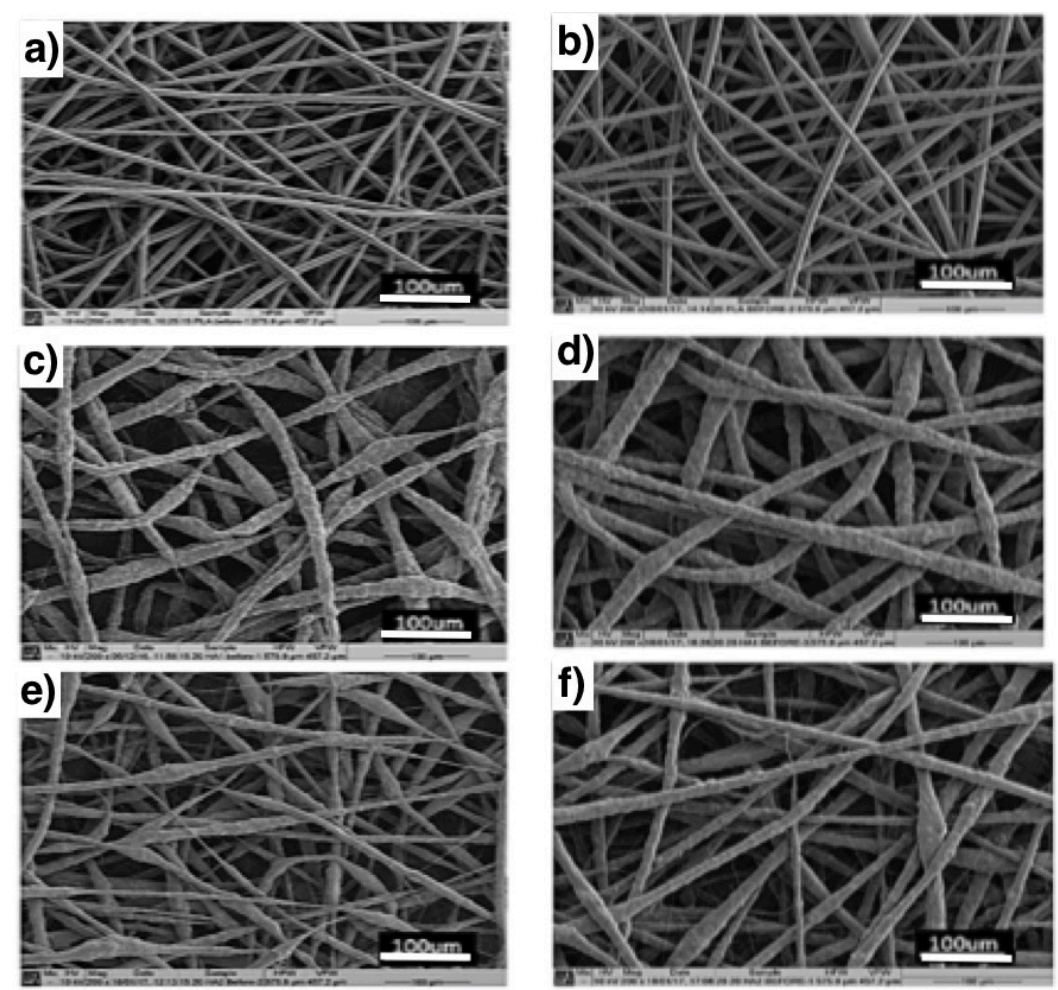

Figure 1 SEM images of electrospun 15\% and 20\% PLA with (a) and (b) no filler, (c) and (d) 20\% HA1 and (e) and (f) 20\% HA2 (marker bars= $100 \mu \mathrm{m}$ )

Table 1 Fibre diameter, porosity and apparent density of electrospun PLA and PLA/HA composite scaffolds

\begin{tabular}{|c|c|c|c|c|c|c|}
\hline \multirow{2}{*}{$\begin{array}{c}\text { Filler } \\
\text { percentage }\end{array}$} & $\begin{array}{c}\text { Fibre } \\
\text { diameter } \\
(\mu \mathrm{m})\end{array}$ & $\begin{array}{c}\text { Porosity } \\
(\%)\end{array}$ & $\begin{array}{c}\text { Density } \\
\left(\mathrm{g} \mathrm{cm}^{-3}\right)\end{array}$ & $\begin{array}{c}\text { Fibre } \\
\text { diameter } \\
(\mu \mathrm{m})\end{array}$ & $\begin{array}{c}\text { Porosity } \\
(\%)\end{array}$ & $\begin{array}{c}\text { Density } \\
\left(\mathrm{g} \mathrm{cm}^{-3}\right)\end{array}$ \\
\hline \multirow{2}{*}{$0 \%$} & $9.28 \pm$ & $90.5 \pm$ & $0.124 \pm$ & $11.16 \pm$ & $91.4 \pm$ & $0.117 \pm$ \\
& 1.64 & 1.2 & 0.015 & 1.66 & 2.9 & 0.025 \\
\hline \multirow{2}{*}{$10 \% \mathrm{HA} 1$} & $20.04 \pm$ & $87.9 \pm$ & $0.174 \pm$ & $19.96 \pm$ & $89.4 \pm$ & $0.151 \pm$ \\
& 6.03 & 2.9 & 0.042 & 5.33 & 2.0 & 0.010 \\
\hline $20 \% \mathrm{HA} 1$ & $20.63 \pm$ & $90.3 \pm$ & $0.157 \pm$ & $15.77 \pm$ & $88.3 \pm$ & $0.189 \pm$ \\
& 8.74 & 1.7 & 0.019 & 4.23 & 0.8 & 0.011 \\
\hline $10 \% \mathrm{HA} 2$ & $20.21 \pm$ & $89.1 \pm$ & $0.156 \pm$ & $20.48 \pm$ & $87.9 \pm$ & $0.173 \pm$ \\
& 5.99 & 0.9 & 0.013 & 5.12 & 1.7 & 0.005 \\
\hline $20 \% \mathrm{HA} 2$ & $18.43 \pm$ & $89.5 \pm$ & $0.170 \pm$ & $18.16 \pm$ & $88.2 \pm$ & $0.190 \pm$ \\
& 7.31 & 2.7 & 0.047 & 6.19 & 0.4 & 0.027 \\
\hline
\end{tabular}

Figure 2 shows the morphologies of electrospun scaffolds after 28 days of SBF immersion. For non-filled PLA scaffolds, the fibres maintained their shape and no swelling or ruptured fibres were noticed. Most of $20 \%$ PLA scaffolds filled with either HA1 or HA2 have also maintained their structure. However, at higher magnification, a number of ruptured or broken fibres were noticed in some 15\% PLA samples and 
apatite precipitation was observed on the fibre surface of both HA1 and HA2 filled scaffolds after immersion (Figure 3).
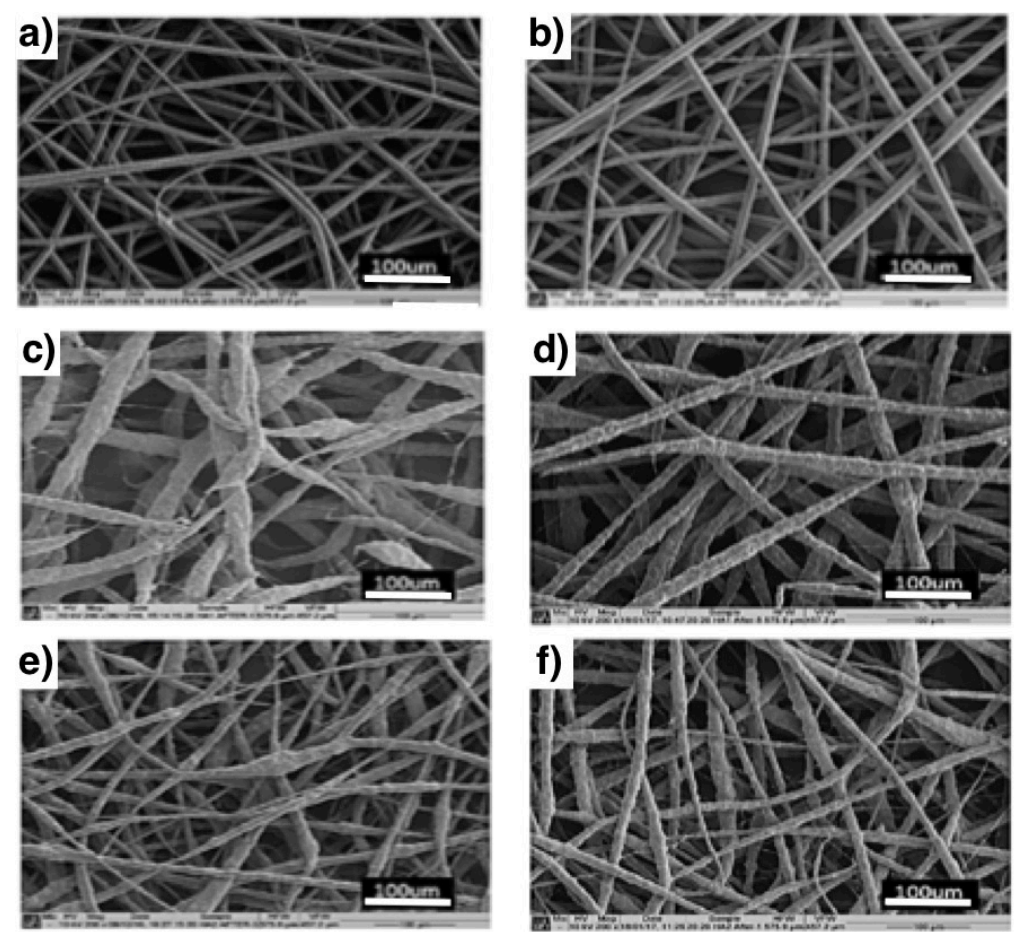

Figure 2 SEM images of electrospun 15\% and 20\% PLA with (a) and (b) no filler, (c) and (d) $20 \%$ HA1 and (e) and (f) $20 \%$ HA2 after immersion in SBF for 28 days (marker bars $=100 \mu \mathrm{m})$
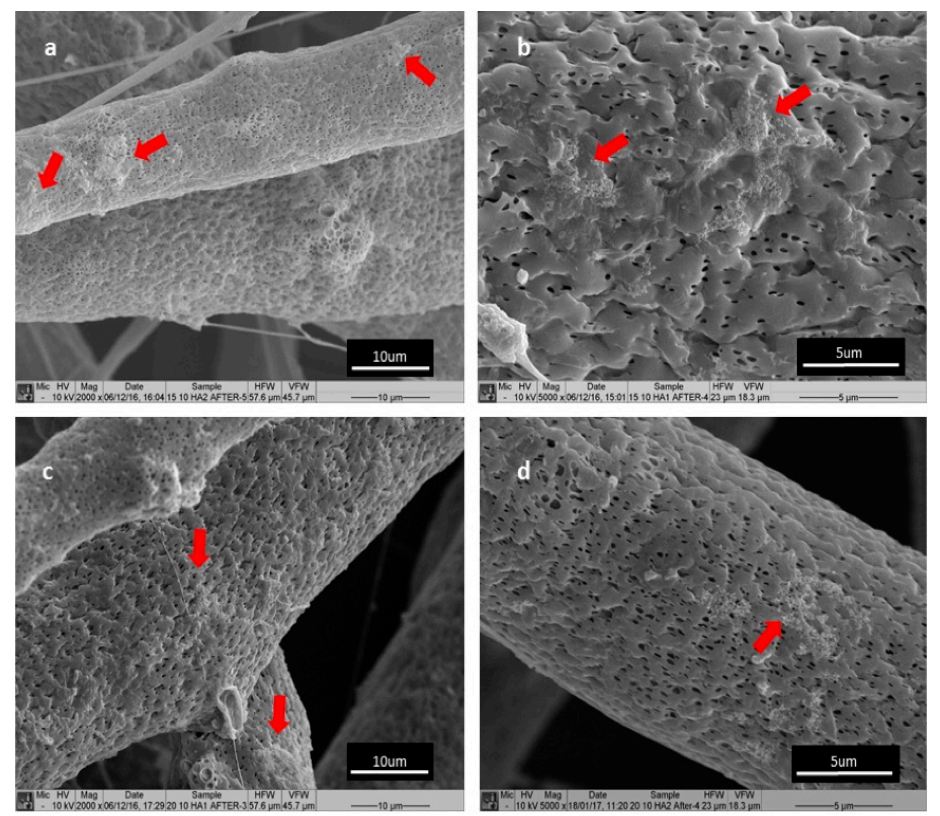

Figure $3 \quad$ Higher magnification SEM images for electrospun (a) 15\% PLA with 10\% HA2, (b) 15\% PLA with 10\% HA1, (c) 20\% PLA with 10\% HA1, and (d) 20\% PLA with $10 \%$ HA2 scaffolds after immersion in SBF for 28 days showing HCA formation on the surface of the fibres (marker bars for $(a)$ and $(c)=10 \mu \mathrm{m}$, and for $(b)$ and $(d)=5 \mu \mathrm{m}$ ) 


\subsection{Water Uptake and Dissolution Rate}

The water uptake and dry weight loss for non-filled and HA-filled PLA scaffolds over 1 , 2, 4, 7, 14, 21 and 28 days of immersion are shown in Figure 4. HA-filled scaffolds showed significantly higher water absorption rates than non-filled PLA scaffolds. However, both non-filled and HA-filled scaffolds showed gradual increases in the water uptake.

Additionally, both non-filled and HA-filled scaffolds exhibited very slow degradation behaviour during 28 days of SBF incubation. The total weight loss for non-filled 15 and $20 \%$ PLA scaffolds were only $3.73 \%$ and $2.79 \%$, respectively. Scaffolds filled with either HA exhibited slower mass loss compared to non-filled PLA scaffolds.

With 15\% PLA scaffolds, the variations between non-filled and HA1-filled scaffolds started to become significant from day 14 of immersion for both 10 and 20\% HA1 scaffolds, while significant differences were only observed between non-filled and HA2-filled scaffolds from day 21 . The rate of mass loss was significantly lower in $15 \%$ PLA scaffolds filled with HA1 than in those filled with HA2. By increasing the PLA concentration to $20 \%$, the difference in mass loss percentage between HA1 and HA2filled scaffolds became generally minor. Increasing filler concentration from 10 to $20 \%$ led to further decreases in mass loss rate, but the effect was not significant, however, at $20 \mathrm{vol} \%$, it significantly increased the variations between the two HAs scaffolds.
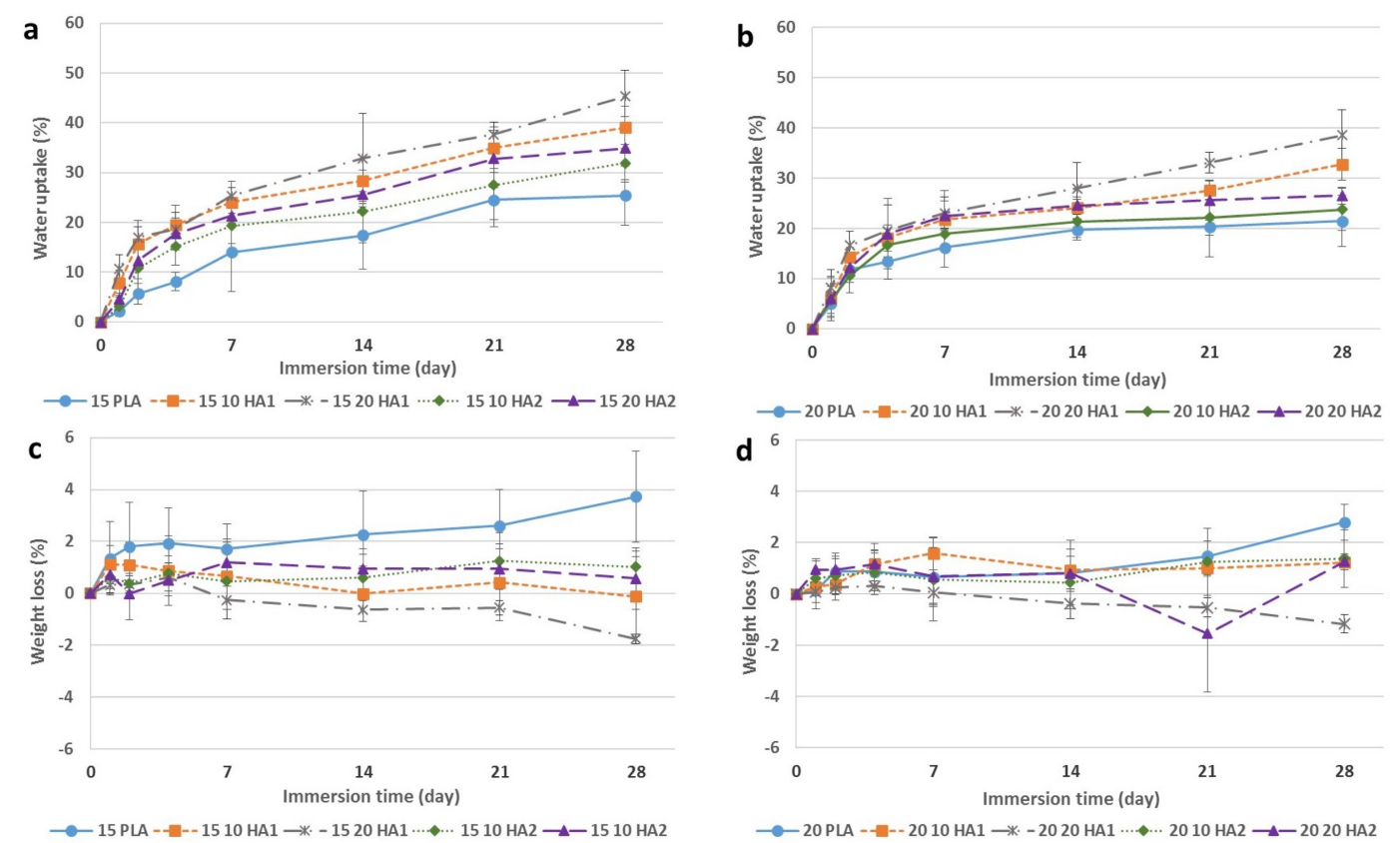

Figure 4 Water uptake ( $a$ and $b$ ) and weight loss ( $c$ and $d$ ) of 15\% PLA samples and $20 \%$ PLA samples with no filler or with 10 or $20 \%$ of HA1 or HA2 during 28 days of immersion in SBF

\subsection{Mechanical testing}

The Young's moduli and ultimate tensile strengths for $15 \%$ and $20 \%$ PLA concentration with 10 or $20 \%$ HA1 or HA2 before and after two weeks immersion in SBF are shown in Figures 5 and 6, respectively. Scaffolds filled with either HA1 or HA2 showed faster deterioration in their strength and stiffness compared to non-filled 15 or 20\% PLA scaffolds. By day 28 the samples were too fragile to test. 


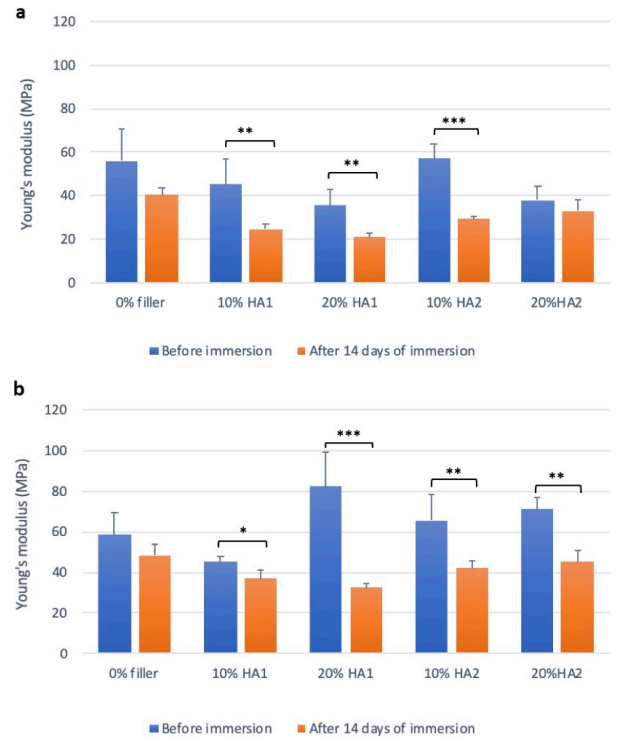

Figure 5 Young's moduli for (a) 15\% PLA, and (b) 20\% PLA samples with no filler or 10 or $20 \%$ of HA1 or HA2 before and after 14 days of immersion in SBF. $\left(^{*}=p<0.05, * *=p<0.01, * * *\right.$ $=p<0.001)$
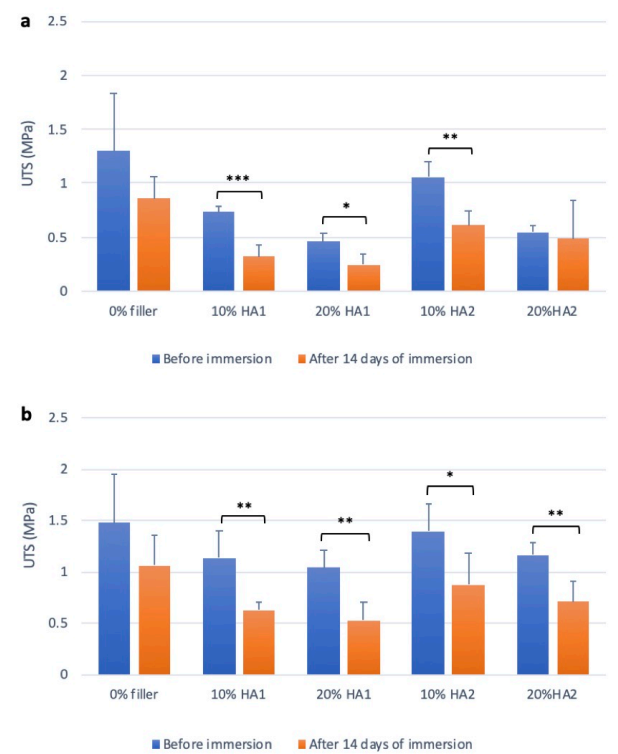

Figure 6

UTS values for (a) $15 \%$ PLA, and (b) 20\% PLA samples with no filler or 10 or $20 \%$ of $\mathrm{HA} 1$ or HA2 before and after 14 days of immersion in SBF. $1^{*}=\mathrm{p}<0.05, * *=p<0.01,{ }^{* * *}=p<$ $0.001)$

\subsection{TGA}

Figures 7 and 8 display the thermogravimetric analysis of non-filled PLA and HA1 or HA2-filled scaffolds before and after 28 days of immersion in SBF. It should be noted that the TGA graphs and the residue percentage are presented in weight\%, with 10 and 20 vol \% HA being equal to 22.1 and 38.9 wt\%, respectively. 

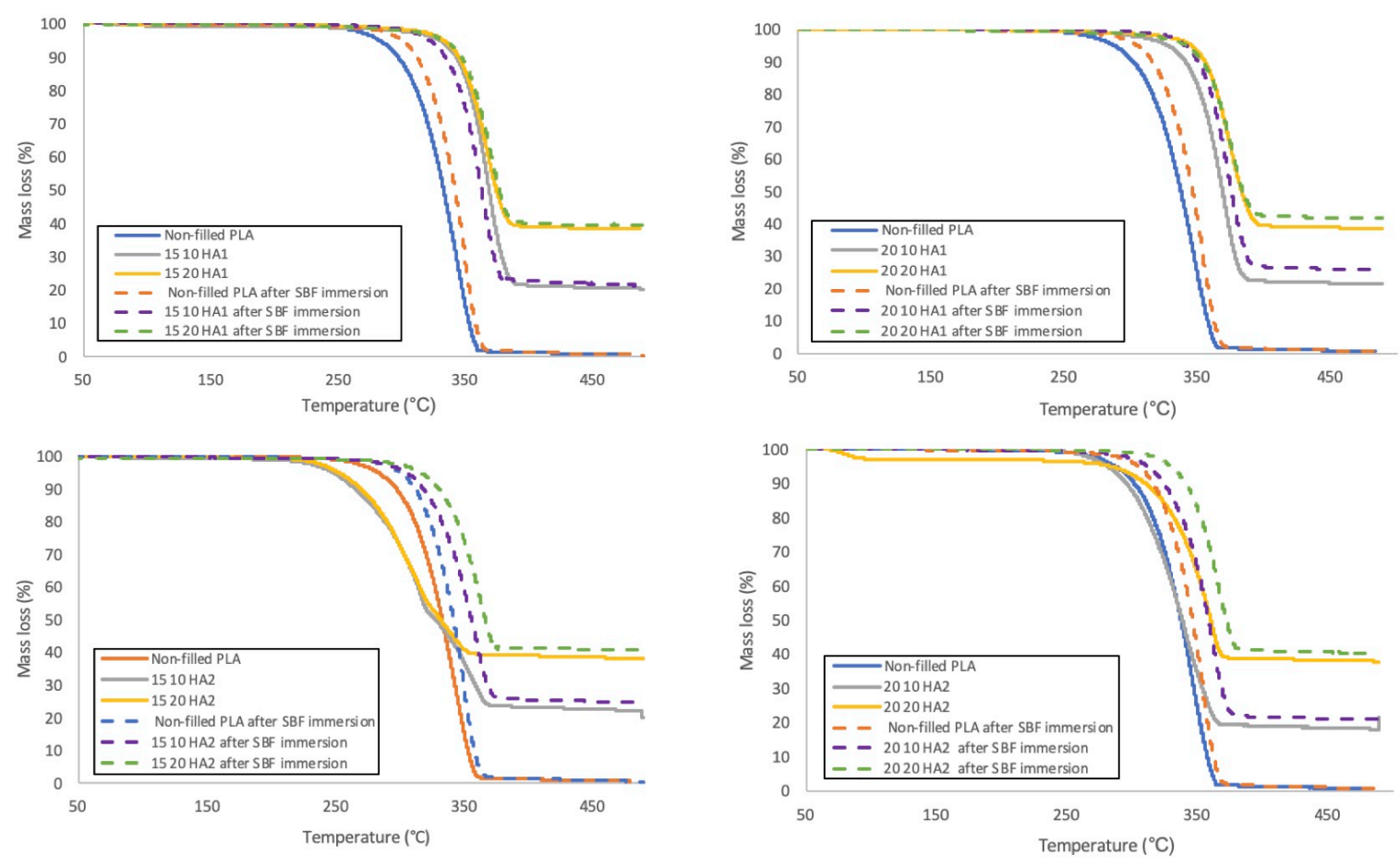

Figure $7 \quad$ TGA curves of non-filled

Figure $8 \quad$ TGA curves of non-filled 15\% PLA scaffolds with (a) HA1 filled and (b) HA2-filled scaffolds before and after SBF immersion for 28 days

20\% PLA scaffolds with (a) HA1 filled and (b) HA2-filled scaffolds before and after SBF immersion for 28 days.

\section{DISCUSSION}

The porosity of biomaterial scaffolds plays a significant role in bone formation in vitro and in vivo. Open porous surfaces and interconnected networks are crucial for tissue vascularization and cell proliferation, nutrition and migration to form new tissues. They also help to facilitate mechanical interlocking between the implant and the surrounding natural bone, which helps increase the mechanical stability at this critical interface $[18,19]$. Table 1 shows that all the scaffolds exhibited satisfactorily high porosity even when the HA content was raised to $20 \%$ and no significant differences were observed between filled and non-filled scaffolds or between scaffolds containing HA1 and those containing HA2.

The surface properties of the implant, including surface roughness, chemistry, topography and energy/wettability influence the initial cellular response at the cellmaterial interface, ultimately affecting the new tissue formation [20, 21]. Figure 1 shows non-filled 15 and 20\% PLA scaffolds before degradation. Both scaffolds exhibit uniform fibre diameter and relatively smooth surfaces, however, incorporation of either HA1 or HA2 usually increased the surface roughness of the composite fibres as well as increasing fibre diameter and irregularity. Visually the fibre roughness was increased by increasing the filler content, while adding HA2 increased fibre diameter slightly, which might be due to agglomerate formation inside the fibres. However, no apparent change in fibre diameter was observed upon increasing HA1 content. Only few protuberances on the fibre surfaces were noticed upon including either HA. Similar results of increased surface roughness upon incorporation of $\mathrm{HA}$ and graphene oxide nanoparticles was reported by Ma et al. [22]. Deng et al. [23] also reported the 
roughness of the PLLA/HA hybrid scaffolds, although their average fibre diameter did not change significantly. In addition, it was also noticed that some fibres were discontinuous or ruptured which might be resulted from needle clogging due to the HA particles which may have caused jet disruption.

High densities of nanopores were observed at the surface of both filled and non-filled electrospun PLA fibres. The pores were elliptical in shape and in the range of 200-350 $\mathrm{nm}$ in length for non-filled PLA scaffolds and from $250 \mathrm{~nm}-1 \mu \mathrm{m}$ for PLA/HA scaffolds, with the longer dimension along with the fibre axis. Pores were larger where the HA particles were closer to the surface of the fibres (Figure 9).
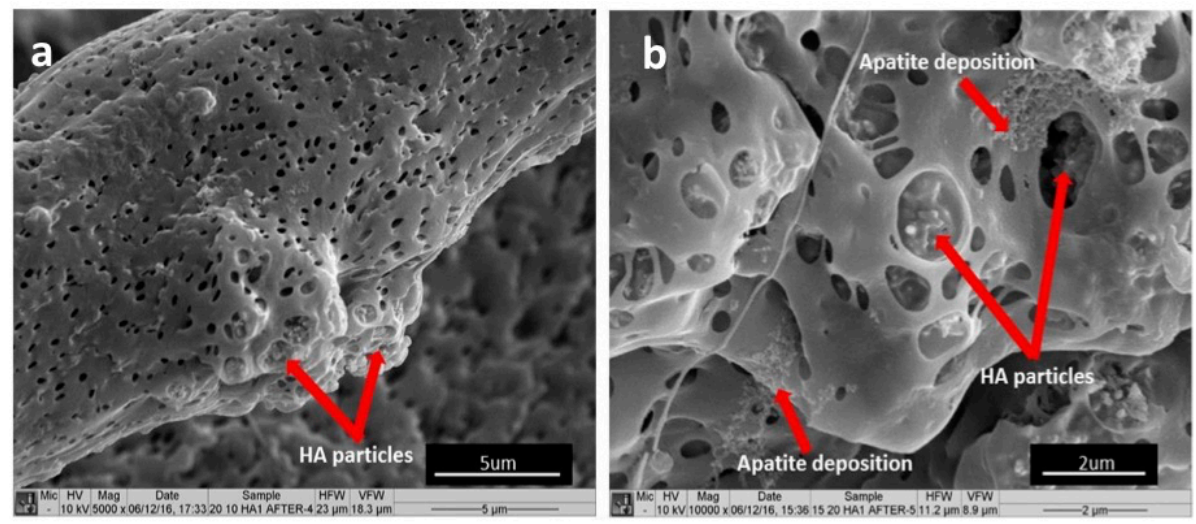

Figure 9 SEM images of (a) 20\% PLA with 10\% HA1 and (b) 15\% PLA with 20\% HA1 scaffolds showing the nanoporous structure of the fibre surface and HA particles inside the fibres (marker bar for $(a)=5 \mu \mathrm{m}$ and for $(b)=2 \mu \mathrm{m}$ ).

Pore formation occurs as a result of using highly volatile solvents such as chloroform or dichloromethane. Two explanations have been presented: according to Bognitzki et al. [24], fast evaporation of the solvent give rise to local phase separation where two phases are formed in the polymeric solution: one that is solvent-rich and the other is solvent-poor. Ultimately, the solvent-rich regions transform into pores during the electrospinning.

Srinivasarao et al. [25] presented a different mechanism for the formation of these surface pores called "breath figures". They described them as imprints created as a result of evaporative cooling during rapid evaporation of the solvent, therefore significantly cooling the surface of the electrospinning jet as it travels from the needle tip to the collector. As the jet surface cools, moisture in the air condenses and grows in the form of minute droplets that act as hard spheres due to convection currents on the surface of the jet. Finally, the water droplets evaporate as the jet dries on the collector, leaving imprints on the surface of the electrospun fibres in the form of pores. Thus, the formation of breath figures requires a certain level of humidity in addition to the use of volatile solvents. Unfortunately, the humidity in the materials preparation laboratory was not measured, but was assumed to be relatively high.

The presence of a porous structure at the fibre surface has several advantages including increasing the surface area, providing of binding sites for drug loading and providing a texture for cell attachment. Finally, the porous structure can affect the 
roughness and wetting behaviour of the scaffolds, as well as specific adsorption processes [26,27].

Mineral deposition is a major property governing bone bioactivity [4]. SEM images of non-filled 15 and 20\% PLA scaffolds showed no significant morphological changes after 4 weeks of immersion in SBF. Fibres of both scaffolds maintained their shape with no significant swelling or reduction in fibre diameter. In addition, no apatite formation was observed on the surface of the fibres, indicating poor mineralisation ability of the plain PLA fibres. This can be attributed to the fact that PLA is hydrophobic and cannot provide enough active functional groups for the formation and growth of a mineralised HA layer. This finding is in agreement with Zhang et al. [28] and Fu et al. [29] who showed that the morphology of PLA fibres had no obvious changes after soaking in SBF for 1, 3, or 7 days, and the surfaces of PLA nanofibers were still smooth after incubation. However, Wang et al. [30] reported increased surface roughness and the formation of plate-like structures and flaky features on the surface of electrospun PLA fibres after immersion in SBF for 7,14 and 21 days at $10^{\circ} \mathrm{C}$, but such structures were not observed in this study.

Morphological analysis of PLA/HA1 and PLA/HA2 scaffolds indicate formation of an apatite layer on the surface of electrospun fibres after 28 days immersion in SBF as shown in Figure 3. Additionally, an increased number of ruptured fibres were noticed after immersion in SBF since the thin regions along those fibres would represent weak points during hydrolysis. Ruptured or broken fibres were rarely observed in non-filled 15 or $20 \%$ PLA scaffolds.

Although scaffolds containing either HA indicated higher in vitro bioactivity than nonfilled scaffolds, the rate of apatite formation was generally lower than expected and the apatite layer did not cover the whole surface of the fibres as reported previously [31-34]. There are three potential causes for the differences in apatite formation rate; the first related with the size of HA particles used in this study. Calcium phosphate bioactivity is believed to be associated with the rate of material dissolution as well as the release of calcium and phosphate ions from the surface of the implant followed by the precipitation of a biological apatite layer [35]. The previously mentioned studies incorporated nano-sized HA within their electrospun scaffolds instead of micro HA. Decreasing the size of HA particle results in larger surface area exposed within the media which may have increased the dissolution of $\mathrm{Ca}$ and $\mathrm{P}$ ions leading to greater apatite deposition with subsequently more protein adsorption, osteoblast adhesion and increased bone growth [36, 37]. Another possible reason for the decreased deposition rate compared to the previous studies is the differences in fibre diameter of the obtained electrospun scaffolds. Increased fibre diameter may reduce the rate of mineralisation in SBF. According to He et al. [38], all fibres are exposed to the same non-accelerated deposition conditions (ionic strength, $\mathrm{pH}$, etc.) during SBF incubation and the nucleation sites compete equally for calcium and phosphate ions, which result in a slower overall deposition rate. In addition, smaller fibres provide a larger relative surface area than larger fibres, potentially leading to faster mineral deposition. Lastly, it is also worth noting that while the complete encapsulation of HA particles within the fibre can enhance the mechanical stability of the resulting scaffold, the degree of bioactivity might be reduced due to the absence of bioactive particles on the fibre surfaces. The presence of HA particles on the surface may release more $\mathrm{Ca}$ and $\mathrm{P}$ ions to the SBF solution, thus inducing more bone-like mineral deposition on the fibre 
surface (Figure I). Kareem et al. [39] showed that partial encapsulation of HA particles in their core and shell PCL-PLA/HA electrospun scaffolds led to significant increases in the rate of apatite precipitation on coaxial fibres during SBF immersion due to more nucleation sites on the fibre surface. Non-filled 15 and 20\% PLA scaffolds showed minimal mass loss rate during four weeks immersion in SBF. Similar results were obtained by You et al. [40], where they noticed no significant weight loss in electrospun PLA scaffolds after 45 days immersion in PBS at $37^{\circ} \mathrm{C}$.

The degradation of PLA under aqueous conditions generally occurs in two stages: the first includes water diffusion into the amorphous regions of the polymer matrix. Once the amorphous regions are degraded, the second stage of degradation starts as a hydrolytic attack proceeding from the edge toward the centre of the crystalline domains [41]. Thus, the hydrolysis rate of PLA electrospun fibres is strongly influenced by polymer crystallinity, which depends on the composition of the polymer chains (the relative amounts of $L$ and $D L$ units), in addition to molecular weight and morphological structure [40]. The fibre diameter may also have a role in the degradation rates, Cui et al. (2006) compared the in vitro degradation of $5 \%$ paracetamol-loaded electrospun PDLLA fibres with average diameters of $212 \mathrm{~nm}, 551 \mathrm{~nm}$, and $1.31 \mu \mathrm{m}$ with that of 100 $\mu \mathrm{m}$ thick PDLLA cast film. After incubation in the degradation medium, the electrospun PDLLA fibre diameter increased and inter fibre spaces decreased. Electrospun mats with an average fibre diameter of $212 \mathrm{~nm}$ lost $18 \%$ of their mass in 9 weeks, whereas cast film and $1.31 \mu \mathrm{m}$ diameter microfibrous electrospun mat lost only $8 \%$.

The addition of HA1 or HA2 particles decreased the mass loss rate significantly compared to plain PLA mats. This can be attributed to the dissolution of alkaline HA particles and the release of $\mathrm{OH}^{-}$ions into the SBF which can neutralize the acidic degradable substances generated during the PLA degradation process. As a result, acid driven autocatalytic degradation of the polymer phase can be reduced, thus slowing degradation. HA incorporation thus can reduce or even prevent aseptic inflammation caused by acid release and thus improve the biocompatibility. This effect of HA slowing down the degradation rate of PLA scaffolds has been reported previously [17, 43, 44].

15\% PLA scaffolds filled with HA1 exhibited significantly lower mass loss rate than scaffolds filled with HA2. This difference is possibly due to the higher surface area of HA1 particles which can increase the ion release compared to HA2 particles. However, the differences in mass loss between the two HAs scaffolds were reduced upon increasing the polymer concentration to $20 \%$, which indicates that polymer concentration can have an impact on the mass loss rate for HA-filled scaffolds, possibly by enhancing the HA encapsulation within the scaffolds, thus reducing their exposure to SBF.

Despite the reduced mass loss rate, HA-filled scaffolds had significantly higher water absorption than non-filled PLA scaffolds. The observed increase in water absorption among HA-filled scaffolds could be attributed to the presence of free and reactive hydroxyl groups on the HA particles which exhibit good affinity to water molecules [45]. Secondly, there are micro-voids in the interface between PLA and HA particles caused by the difference in the chemical nature of PLA and HA or due to non-uniform distribution of HA particles along the fibres. Thus, water molecules can penetrate the micro-voids and accumulate at the PLA/HA interface, increasing the water absorption. These results are consistent with those of Rong et al. [46] and Huang et al. [44] who 
found that incorporation of HA particles into scaffolds resulted in increased water absorption while decreasing the mass loss rate. However, Sui et al. [43] found that introduction of HA particles decreased both mass loss and water uptake of PLA/HA scaffolds. They attributed the reduced water uptake to the dissolution of alkaline HA particles, blocking water uptake.

Scaffolds containing HA1 exhibited higher water absorption rates than HA2-filled scaffolds. This is probably due to the higher surface area of HA1 particles which can increase water absorption by HA particles and penetration by increasing the cavities at the PLA/filler interface. The major difference between the two HA grades is the specific surface area, which is an order of magnitude different. Thus it may be assumed that the larger surface area needs more matrix to cover the particle surface completely [47], and consequently for the same amount of polymer matrix, larger voids can be created around the HA1 particles compared HA2 particles as there is no external force added during the electrospinning to force the polymer matrix to fill the PLA/HA interface and cover the whole HA particles, which leads to increased potential for water accumulation in HA1-filled scaffolds.

According to Suwanprateeb et al. [48] the degree of water absorption in nondegradable polymers depends on both filler content and the length of immersion. The higher the filler content, the more liquid which can diffuse into the composite due to the greater number of penetration sites and higher interface area. In this study, increasing HA1 or HA2 content from 10 to $20 \%$ vol resulted in slight, albeit nonsignificant, increases in water absorption for both 15 and 20\% PLA scaffolds.

Biodegradation and tissue ingrowth have major effects on the mechanical and structural properties of implanted devices. Therefore, in vitro evaluation of the mechanical properties of biomaterials composites is important [49,50]. Mechanical properties of non-filled PLA and PLA/HA scaffolds were evaluated after 14 days of soaking in SBF (Figures 5 and 6). All scaffolds lost stiffness and strength after immersion in SBF, however, the reduction in non-filled 15 and 20\% PLA scaffolds were not significant for either UTS or Young's modulus. On the other hand, scaffolds containing either type of HA showed greater reduction in tensile strength and modulus compared to non-filled scaffolds, probably due to the increased moisture absorption of HA-filled scaffolds, reducing the interfacial adhesion between the filler and the matrix. A further decrease was seen in the mechanical properties of HA1 containing samples compared to HA2 containing samples, which could be due to the larger voids at the PLA/HA1 interface and the higher moisture absorption of HA1 filled scaffolds which results in faster interface debonding, thereby reducing the mechanical stability. However, the differences between the samples containing the two HAs became less significant when PLA concentration was increased to $20 \%$ as differences in the water absorption rates reduced. Furthermore, results have shown large variations in stiffness and strength values among 15 and 20\% PLA scaffolds containing 10 and 20 vol\% of HA1 or HA2 after immersion in SBF. It should be noted that in addition to the effect of filler content, other factors such as fibre discontinuity, fusion and packing density, filler agglomeration within the fibres, formation of nano/micro cracks in the fibres during scaffolds processing can all affect the mechanical properties of the scaffolds.

Thermogravimetric analysis has been used extensively to examine the thermal stability and decomposition of PLA and PLA based composites and to determine the 
amount of inorganic filler incorporated in the scaffolds [51, 52]. In this study, TGA was also used to measure the amount of apatite formed on the fibre surfaces after immersion in SBF. Figures 7 and 8 showed that the mass percentages of HA particles incorporated in HA1 or HA2-filled scaffolds were consistent with the actual amount of $\mathrm{HA}$ added into the polymer solutions before electrospinning. Since HA has a higher density than PLA ( 3.162 vs $1.24 \mathrm{~g} \mathrm{~cm}^{-3}$ ), the precipitation of HA particles in PLA solutions can easily occur especially when high percentages of HA are used, which may reduce the total amount of HA within the electrospun fibres. TGA results indicated, however, that the solutions were electrospun with minimal loss of HA. Scaffolds filled with either HA showed increased residue weight after immersion in SBF confirming apatite formation on the fibre surfaces. However, increasing the filler surface area or filler content in the scaffolds did not appear to have a significant impact on the amount of apatite formed after immersion. These observations agree with SEM and weight loss results which indicated apatite formation on HA-filled scaffolds but slowly.

Scaffolds with HA1 showed an increase in the thermal stability compared to non-filled 15 and 20\% PLA scaffolds. In contrast, the thermal stability of HA2-filled scaffolds at 15\% PLA concentration were surprisingly lower than both non-filled and HA1-filled scaffolds which could be due to higher exposure of $\mathrm{OH}$ groups on $\mathrm{HA}$ surface to the PLA matrix, leading to formation of some agglomerates within the fibres, thus reducing the thermal stability [54]. However, scaffolds with 20\% PLA concentration gave higher onset temperatures than non-filled scaffolds which might be due to better dispersion of HA2 within the polymeric matrix, but was still lower than HA1-filled scaffolds. According to Ignjatovic et al. [53] and Rakmae et al. [54], there are two potential reasons for the enhanced thermal stability of polymer composites: either the well dispersed filler provides good adhesion with the matrix and blocks polymer decomposition products or the filler particles act as a barrier preventing heat transfer. Thus, it seems logical that HA1 can provide higher thermal stability than HA2 due to its higher surface area which can provide better insulation to PLA against thermal degradation.

FTIR results of scaffolds containing both types of HAs showed additional peaks at 468, $560,602,630$, and $1022 \mathrm{~cm}^{-1}$ compared to plain PLA scaffolds, which belong to the $\mathrm{OH}^{-1}$ and $\mathrm{PO}^{-3}$ bands of $\mathrm{HA}$. Sui et al. [43] reported development of a new peak assigned to $\mathrm{COO}^{-}$at $1600 \mathrm{~cm}^{-1}$ as well as formation of a weak $\mathrm{OH}$ peak around 3500 $\mathrm{cm}^{-1}$ in the FTIR spectra of electrospun PLLA/HA scaffolds as a result of the interaction between $\mathrm{COOH}$ in PLLA and $\mathrm{Ca}^{2+}$ in $\mathrm{HA}$. However, no additional peaks were present in the PLA/HA spectra obtained in this study, confirming solely mechanical incorporation of HA into the polymer phase without chemical bonding. The intensities of HA-specific bands in the composite spectrum were in agreement with the amounts of HA incorporated in the scaffolds.

Previous studies reported a significant increase of HA-specific bands intensities due to apatite formation on the fibre surface after few days of SBF immersion in addition to the higher intensity and lower width at half maximum of the $\mathrm{C}=\mathrm{O}$ band at $1759 \mathrm{~cm}^{-1}$ due to polymer degradation [33, 55, 56]. Interestingly, no significant changes appeared on either non-filled and HA-filled scaffolds spectra after 28 days of SBF immersion except for very small increases in the intensity of 560 and $630 \mathrm{~cm}^{-1}$ peaks in HA-filled samples which were assigned to the phosphate group of hydroxyapatite which suggest the formation of apatite layer on the surface of the samples. However, 
by comparing these results to SEM, weight loss and TGA results, a significant variation in FTIR results before and after immersion would not be expected due to low degradation and limited bioactivity rate of samples. Additionally, detection of the precipitated apatite peaks would be challenging due to the high penetration depth of FTIR energy into the samples $(0.5-5 \mu \mathrm{m})$ compared to the thickness of apatite layer formed on the scaffolds surfaces.

\section{CONCLUSIONS}

PLA dissolved in chloroform at concentrations between 15 and 20 wt\% produced uniform non-woven electrospun fibres. Introducing either of the HAs into electrospun PLA scaffolds led to increased fibre diameter and surface roughness compared to plain PLA scaffolds. It also reduced degradation and increased in vitro bioactivity during immersion in SBF. However, increasing filler content or filler surface area did not affect the porosity.

Mechanical properties of non-filled and HA-filled scaffolds reduced significantly after 14 days of immersion in SBF, while they were too fragile to test at day 28 of immersion. HA1filled scaffolds exhibited faster declines in their tensile strength than HA2-filled scaffolds probably due to the larger specific surface area of HA1 which permits increased water penetration through the cavities created at PLA/HA interface, while differences in the degradation and apatite formation rates from using the two different types of HAs were generally minor. In contrast, scaffolds containing HA1 exhibited enhanced thermal stability over HA2-filled scaffolds due to higher surface area of the HA1 particles.

\section{ACKNOWLEDGEMENTS}

The work was supported by an Iraqi Government Scholarship Grant (number S1648). The authors would also like to thank Dr Margaret Smith, Mrs Margaret Mullin and Mr John Davidson for their help with FTIR, SEM and mechanical testing, respectively.

\section{REFERENCES}

1. Drew C, Wang X, Samuelson LA, Kumar J. The Effect of Viscosity and Filler on Electrospun Fiber Morphology. J Macromol Sci Part A.; 2003;40:1415-22.

2. Tyagi P, Catledge SA, Stanishevsky A, Thomas V, Vohra YK. Nanomechanical properties of electrospun composite scaffolds based on polycaprolactone and hydroxyapatite. J Nanosci Nanotechnol. 2009;9:4839-45.

3. Kouhi M, Prabhakaran MP, Shamanian M, Fathi M, Morshed M, Ramakrishna S. Electrospun PHBV nanofibers containing $\mathrm{HA}$ and bredigite nanoparticles: Fabrication, characterization and evaluation of mechanical properties and bioactivity. Compos Sci Technol.; 2015;121:115-22.

4. Lao L, Wang Y, Zhu Y, Zhang Y, Gao C. Poly(lactide-co-glycolide)/hydroxyapatite nanofibrous scaffolds fabricated by electrospinning for bone tissue engineering. J Mater Sci Mater Med. 2011;22:1873-84.

5. Zhang Y, Venugopal JR, El-Turki A, Ramakrishna S, Su B, Lim CT. Electrospun biomimetic nanocomposite nanofibers of hydroxyapatite/chitosan for bone tissue engineering. Biomaterials. 2008;29:4314-22.

6. Peng $\mathrm{F}, \mathrm{Yu} \mathrm{X}$, Wei M. In vitro cell performance on hydroxyapatite particles/poly(L-lactic acid) nanofibrous scaffolds with an excellent particle along nanofiber orientation. Acta Biomater.; 2011;7:2585-92.

7. Tetteh G, Khan AS, Delaine-Smith RM, Reilly GC, Rehman IU. Electrospun polyurethane/hydroxyapatite bioactive scaffolds for bone tissue engineering: The role of solvent and hydroxyapatite particles. J Mech Behav Biomed Mater. 2014;39:95-110.

8. Yang S, Madbouly SA, Schrader JA, Srinivasan G, Grewell D, McCabe KG, et al. Characterization and biodegradation behavior of bio-based poly(lactic acid) and soy protein blends for sustainable 
horticultural applications. Green Chem.; 2015;17:380-93.

9. Ma Z, Kotaki M, Yong T, He W, Ramakrishna S. Surface engineering of electrospun polyethylene terephthalate (PET) nanofibers towards development of a new material for blood vessel engineering. Biomaterials. 2005;26:2527-36.

10. He W, Ma Z, Yong T, Teo WE, Ramakrishna S. Fabrication of collagen-coated biodegradable polymer nanofiber mesh and its potential for endothelial cells growth. Biomaterials. 2005;26:7606-15.

11. Guarino V, Causa F, Taddei P, di Foggia M, Ciapetti G, Martini D, et al. Polylactic acid fibre-reinforced polycaprolactone scaffolds for bone tissue engineering. Biomaterials. 2008;29:3662-70.

12. Whelan T. Polymer Technology Dictionary. London: Chapman \& Hall; 1994.

13. Kokubo T, Kushitani H, Sakka S, Kitsugi T, Yamamuro T. Solutions able to reproduce in vivo surfacestructure changes in bioactive glass-ceramic A-W3. J Biomed Mater Res. 1990;24:721-34.

14. Oyane A, Kim H-M, Furuya T, Kokubo T, Miyazaki T, Nakamura T. Preparation and assessment of revised simulated body fluids. J Biomed Mater Res. 2003;65:188-95.

15. Choi EJ, Son B, Hwang TS, Hwang EH. Increase of degradation and water uptake rate using electrospun star-shaped poly(d,I-lactide) nanofiber. J Ind Eng Chem.; 2011;17:691-5.

16. Augustine R, Thomas S, Kalarikkal N. In Vitro Degradation of Electrospun Polycaprolactone Membranes in Simulated Body Fluid. Int. J. Inst. Mater. Malaysia 2015;2;211-20.

17. Huang J, Xiong J, Liu J, Zhu W, Wang D. Investigation of the in vitro degradation of a novel polylactide/nanohydroxyapatite composite for artificial bone. J Nanomater. 2013;2013.

18. Karageorgiou V, Kaplan D. Porosity of 3D biomaterial scaffolds and osteogenesis. Biomaterials. 2005;26:5474-91.

19. Loh QL, Choong C. Three-dimensional scaffolds for tissue engineering applications: role of porosity and pore size. Tissue Eng Part B Rev. 2013;19:485-502. 20. Feller L, Jadwat Y, Khammissa RAG, Meyerov R, Schechter I, Lemmer J. Cellular responses evoked by different surface characteristics of intraosseous titanium implants. Biomed Res Int. 2015;2015:1-8.

21. Agrawal CM, Ray RB. Biodegradable polymeric scaffolds for musculoskeletal tissue engineering. J Biomed Mater Res. 2001;55:141-50.

22. Ma HB, Su WX, Tai ZX, Sun DF, Yan XB, Liu B, Xue QJ. Preparation and cytocompatibility of polylactic acid/hydroxyapatite/graphene oxide nanocomposite fibrous membrane. Chinese Sci Bull. 2012;57:3051-8.

23. Deng X-L, Sui G, Zhao M-L, Chen G-Q, Yang X-P. Poly(L-lactic acid)/hydroxyapatite hybrid nanofibrous scaffolds prepared by electrospinning. J Biomater Sci Polym Ed.; 2007;18:117-30.

24. Bognitzki M, Czado W, Frese T, Schaper A, Hellwig M, Steinhart M, et al. Nanostructured Fibers via Electrospinning. Adv Mater. 2001;13:70-2.

25. Srinivasarao M, Collings D, Philips A, Patel S. Three-dimensionally ordered array of air bubbles in a polymer film. Science 2001;292(5514):79-83.

26. Kim CH, Jung YH, Kim HY, Lee DR, Dharmaraj N, Choi KE. Effect of collector temperature on the porous structure of electrospun fibers. Macromol Res. 2006;14:59-65.

27. Zander NE. Hierarchically structured electrospun fibers. Polymers. 2013;5:19-44.

28. Zhang H, Fu Q-W, Sun T-W, Chen F, Qi C, Wu J, et al. Amorphous calcium phosphate, hydroxyapatite and poly(D,L-lactic acid) composite nanofibers: Electrospinning preparation, mineralization and in vivo bone defect repair. Colloids Surfaces B-Biointerfaces; 2015;136:27-36.

29. Fu Q-W, Zi Y-P, Xu W, Zhou R, Cai Z-Y, Zheng W-J, et al. Electrospinning of calcium phosphatepoly $(D, L-l a c t i c$ acid $)$ nanofibers for sustained release of water-soluble drug and fast mineralization. Int J Nanomedicine.; 2016;11:5087-97.

30. Wang M, Cai Y, Zhao B, Zhu P. Time-resolved study of nanomorphology and nanomechanic change of early-stage mineralized electrospun poly(lactic acid) fiber by scanning electron microscopy, Raman spectroscopy and atomic force microscopy. Nanomaterials. 2017;7:1-11.

31. Rajzer I. Fabrication of bioactive polycaprolactone/hydroxyapatite scaffolds with final bilayer nano/micro-fibrous structures for tissue engineering application. J Mater Sci. 2014;49:5799-807.

32. Silva CSR, Luz GM, Mano JF, JL Gómez Ribelles, Gómez-Tejedor J. Poly(epsilon-caprolactone) electrospun scaffolds filled with nanoparticles. Production and optimization according to Taguchi's methodology. J Macromol Sci Part B Phys. 2014;53:781-99.

33. Rajzer I, Menaszek E, Kwiatkowski R, Chrzanowski W. Bioactive nanocomposite PLDL/nanohydroxyapatite electrospun membranes for bone tissue engineering. J Mater Sci Mater Med. 2014;25:1239-47.

34. Hassan MI, Sultana N, Hamdan S. Bioactivity assessment of poly ( $\varepsilon$-caprolactone)/ hydroxyapatite 
electrospun fibers for bone tissue engineering application. J Nanomater. 2014;2014.

35. Porter AE, Buckland T, Hing K, Best SM, Bonfield W. The structure of the bond between bone and porous silicon-substituted hydroxyapatite bioceramic implants. J Biomed Mater Res Part A. 2006;78:25-33.

36. Coathup MJ, Cai Q, Campion C, Buckland T, Blunn GW. The effect of particle size on the osteointegration of injectable silicate-substituted calcium phosphate bone substitute materials. J Biomed Mater Res - Part B Appl Biomater. 2013;101 B:902-10.

37. Arsad MSM, Lee PM, Hung LK. Morphology and particle size analysis of hydroxyapatite micro- and nano-particles. CSSR 2010 - 2010 Int Conf Sci Soc Res. 2010. p. 1030-4.

38. He C, Jin X, Ma PX. Calcium phosphate deposition rate, structure and osteoconductivity on electrospun poly(I-lactic acid) matrix using electrodeposition or simulated body fluid incubation. Acta Biomater. 2014;10:419-27.

39. Kareem MM, Hodgkinson T, Sanchez MS, Dalby MJ, Tanner KE. Hybrid core-shell scaffolds for bone tissue engineering. Biomed Mater.; 2019;14; 025008.

40. You Y, Min BM, Lee SJ, Lee TS, Park WH. In vitro degradation behavior of electrospun polyglycolide, polylactide, and poly(lactide-co-glycolide). J Appl Polym Sci. 2005;95:193-200.

41. Dong Y, Liao S, Ngiam M, Chan CK, Ramakrishna S. Degradation Behaviors of Electrospun Resorbable Polyester Nanofibers. Tissue Eng Part B Rev. 2009;15:333-51.

42. Cui W, Li X, Zhu X, Yu G, Zhou S, Weng J. Investigation of drug release and matrix degradation of electrospun poly(DL-lactide) fibers with paracetanol inoculation. Biomacromolecules. 2006;7:1623-9.

43. Sui G, Yang X, Mei F, Hu X, Chen G, Deng X, et al. Poly-L-lactic acid/hydroxyapatite hybrid membrane for bone tissue regeneration. J Biomed Mater Res A. 2007;82A:445-54.

44. Huang J, Xiong J, Liu J, Zhu W, Chen J, Duan L, et al. Evaluation of the novel three-dimensional porous poly (L-lactic acid)/nano-hydroxyapatite composite scaffold. Biomed Mater Eng. 2015;26:S197-205.

45. Tham WL, Chow WS, Ishak ZAM. Simulated body fluid and water absorption effects on poly(methyl methacrylate)/hydroxyapatite denture base composites. Express Polym Lett. 2010;4:517-28.

46. Rong Z, Zeng W, Kuang Y, Zhang J, Liu X, Lu Y, et al. Enhanced bioactivity of osteoblast-like cells on poly(lactic acid)/poly(methyl methacrylate)/nano-hydroxyapatite scaffolds for bone tissue engineering. Fibers Polym. 2015;16:245-53.

47. Joseph R, McGregor WJ, Martyn MT, Tanner KE, Coates PD. Effect of hydroxyapatite morphology/surface area on the rheology and processability of hydroxyapatite filled polyethylene composites. Biomaterials. 2002;23:4295-302.

48. Suwanprateeb J, Tanner KE, Turner S, Bonfield W. Influence of Ringer's solution on creep resistance of hydroxyapatite reinforced polyethylene composites. J Mater Sci Mater Med. 1997;8:469-72.

49. Zhang Y, Tanner KE. Impact behavior of hydroxyapatite reinforced polyethylene composites. J Mater Sci Mater Med. 2003;14:63-8.

50. Krynauw H, Bruchmüller L, Bezuidenhout D, Zilla P, Franz T. Degradation-induced changes of mechanical properties of an electro-spun polyester-urethane scaffold for soft tissue regeneration. J Biomed Mater Res - Part B Appl Biomater. 2011;99 B:359-68.

51. Liu X, Wang T, Chow LC, Yang M, Mitchell JW. Effects of inorganic fillers on the thermal and mechanical properties of poly(lactic acid). Int J Polym Sci. 2014;2014:1-8.

52. Liu X, Khor S, Petinakis E, Yu L, Simon G, Dean K, et al. Effects of hydrophilic fillers on the thermal degradation of poly(lactic acid). Thermochim Acta.; 2010;509:147-51.

53. Ignjatovic N, Suljovrujic E, Budinski-Simendic J, Krakovsky I, Uskokovic D. Evaluation of hot-pressed hydroxyapatite/poly-L-lactide composite biomaterial characteristics. J Biomed Mater Res - Part B Appl Biomater. 2004;71:284-94.

54. Rakmae S, Ruksakulpiwat Y, Sutapun W, Suppakarn N. Physical properties and cytotoxicity of surface-modified bovine bone-based hydroxyapatite/poly(lactic acid) composites. J Compos Mater. 2011;45:1259-69.

55. Sooksaen P, Pengsuwan N, Karawatthanaworrakul S, Pianpraditkul S. Formation of porous apatite layer during in vitro study of hydroxyapatite-AW based glass composites. Adv Condens Matter Phys. 2015;2015:1-9.

56. Chlopek J, Morawska-Chochol A, Paluszkiewicz C, Jaworska J, Kasperczyk J, Dobrzyński P. FTIR and NMR study of poly(lactide-co-glycolide) and hydroxyapatite implant degradation under in vivo conditions. Polym Degrad Stab. 2009;94:1479-85. 